ESAIM: COCV 27 (2021) E3

https://doi.org/10.1051/cocv/2021092
ESAIM: Control, Optimisation and Calculus of Variations

www.esaim-cocv.org

\title{
INTRODUCTION: ON ENRIQUE
}

\author{
Jean-Michel CoRon $^{1, *}$ and Alain Haraux ${ }^{2}$
}

\begin{abstract}
Some of the main contributions of the highly cited mathematician Enrique Zuazua are outlined at the occasion of his sixtieth birthday. Through various anecdotes, it is shown that taking advantage of favourable chances is part of a fruitful career and can produce important results for the benefit of the whole community.
\end{abstract}

Mathematics Subject Classification. 34L20, 35XXX, 49A22, 73K12, 74XXX, 93XXX.

Accepted September 12, 2021.

\section{INTRODUCTION}

In this text, we try to outline some of the main contributions of our colleague Enrique Zuazua at the occasion of his sixtieth birthday. The second author was Enrique's thesis advisor and the first author has followed more thoroughly his achievements in the field of mathematical control theory. A very important part, but not all, of Enrique Zuazua's scientific activity has been devoted to the behavior of waves in a bounded domain: oscillations, stability, stabilization and control. This comes from the fact that after the results obtained in the first part of his thesis with the second author, Enrique was involved in the theory of exact controllability in continuum mechanics, devised after 1986 by Jacques Louis Lions who had in mind some application to the stabilization of large spatial structures. However, to stabilize these structures, some thermal effects have to be taken account of, and the real equations are in fact coupled systems of hyperbolic and parabolic equations. Because parabolic problems are not reversible in time, the controllability of semilinear heat equations has become a field of research in itself, based on quite different methods. The synthesis of parabolic and hyperbolic components is still to come, after a proper modelization for the couplings involved. For the time being, the hyperbolic and parabolic theories are still a source of challenging open problems.

Before entering the main topic of this text, we would like to make some general remarks since the case of our colleague's career is a good example of a longstanding debate opposing "speed" and "depth". The first observation is that in Mathematics, either pure or applied, the time scale is quite different from its analogue in applied Science. We sometimes hear that in Physics, the time of half-obsolescence is about 20 years, while in computer science it is about 2 years. In Mathematics, the relevant time unit is the century rather than years or decades. This might give the impression that "speed" of thinking is not important, while it is exactly the contrary. If a person is "slow" to solve basic problems, when it becomes necessary to overcome several combined difficulties to find the truth, the time scale to solve the problem becomes soon larger than a human life time. So

Keywords and phrases: Wave equations, stability, control theory, spectral analysis.

1 Sorbonne Université, Université de Paris, CNRS, INRIA, Laboratoire Jacques-Louis Lions, équipe Cage, Paris, France.

2 Sorbonne Université, Université de Paris, CNRS, Laboratoire Jacques-Louis Lions, Paris, France.

* Corresponding author: jean.m.coron@gmail.com 
in our field speed is essential and the opposition between speed and depth is fake. We shall see at the beginning of this text that speed of thinking was the first obvious feature of Enrique's behavior. Then he also proved that he can become deep when the solution of problems makes it necessary.

Enrique Zuazua was lucky enough to meet Jacques-Louis Lions and start to collaborate with his powerful team even before finishing his thesis. It was luck in a sense because special circumstances made this possible. But, as we shall explain later, this luck was not a pure effect of chance, and if it was made so productive, it is mainly because curiosity, creativity and persistence were involved in Enrique's approach of the problems. Without these three qualities, luck may become a poisoned gift. The meetings with Enrique typically end up with a sentence of the following type: "well, now let us work this out and then we will see..."

\section{First Realizations, a Report by Alain Haraux}

Enrique started his career at Laboratory Jacques-Louis Lions (At that time called "Laboratoire d'Analyse Numérique") in Paris by preparing a Ph.D. degree under my supervision. As we shall see, the short period devoted to this preparation was already quite productive at several levels. Enrique was one of the brightest students of the Master 2 in Laboratory Jacques-Louis Lions, and was introduced to me by Maria Esteban who came also from Basque country a few years before and held a research position in our department. My first impression was that of a modest and somewhat shy young man, but I understood rather quickly that, as intelligent people usually do, he always observed his interlocutor some time before revealing his true personality.

\subsection{The birth of a sprinter}

In our first meeting, I asked Enrique a simple looking open question which seemed logically solvable in finite time. The question was about the existence of zeroes in a cylinder $(0, T) \times \Omega$ for the solutions of the equation

$$
u_{t t}+\Delta^{2} u=0
$$

in $\mathbb{R} \times \Omega$ (where $\Omega$ is a nonempty bounded domain of $\mathbb{R}^{n}$ for a given positive integer $n$ ) with boundary conditions of homogeneous Dirichlet type $u=\|\nabla u\|=0$ on $\partial \Omega$ for $T$ large enough. Such results were previously known for equations of the form

$$
u^{\prime \prime}+A u=0
$$

and even for some semilinear perturbations when $A$ is a positive self-adjoint operator with compact inverse on $L^{2}(\Omega)$, assuming that the first eigenvalue of $A$ is realized on some positive eigenfunction. For the case of the bi-Laplacian it is known that such eigenfunctions can fail to be of one sign, for instance if $\Omega$ is an annulus. But of course in such a case all solutions with initial state made of multiples of that eigenfunction change sign in any cylinder $(0, T) \times \Omega$, so that it is hard to believe that the sign changes of the first eigenfunction really makes the situation worse. When I asked the question to Enrique, he almost immediately asked whether it would be useful to find a positive supersolution, i.e. a positive solution of

$$
\Delta^{2} \psi \geq \lambda_{1} \psi, \text { for some } \lambda_{1}>0
$$

Then, maybe the next day he told me that a variational principle of G. Stampacchia that he recently learned from the seminar course of Haim Brezis should allow to do that. One week after my question was solved. A few months later we wrote the joint paper [11] containing many more general results, and finally we obtained that indeed, when the first eigenfunction is not of one sign, the global oscillation time becomes smaller than $\frac{\pi}{\sqrt{\lambda_{1}}}$ which is the optimal oscillation time when the first eigenfunction has a constant sign! 


\subsection{Achievements on stability theory of waves}

Some time later, Enrique Zuazua obtained partial results on the oscillatory behavior of solutions to dissipative wave equations when the dissipation is small enough. Then I started to ask him some rather technical questions directly connected with my personal main field of research, something that I almost never did with my students. The questions concerned the extent of stability (rate of decay) of the "permanent regime" (for instance stationary, periodic or almost periodic solution) of dissipative wave equations of the general form

$$
u^{\prime \prime}+L u+g\left(u^{\prime}\right)=h(t, x)
$$

For instance when $g$ is a sum of two different powers, the highest power gives the worst rate of convergence and one would like to prove, under assumptions related to Sobolev inequalities, that the rate of convergence is actually dictated by the lowest power. I had tried to do that for some time and the question became important for me since I was writing the monograph [9] on dissipative hyperbolic problems following an invitation of Jacques-Louis Lions and Jean Dieudonné. In a matter of a few months, Enrique developed new techniques which allowed to do that and even much better. This resulted in two publications, one [10] in collaboration in the simplest case and the other [27] where he was the sole author for more complicated situations. The technique of these papers have been used subsequently by several authors, and to my knowledge the results in the initial setting have never been improved until now, concerning either the results or the hypotheses.

\subsection{Meeting J.L Lions: the decisive impulse}

Chance sometimes plays a role not only for our results, but to guide our careers. In 1986, J.L. Lions, who was delivering his regular course at College de France, asked me whether I could help to write down the corresponding lecture notes. Unfortunately (or maybe fortunately!) I had a long sojourn already planned in Tokyo which made it impossible for me to follow most of his lectures in the fall of 1986. A few weeks before, I discussed with Jesus Hernandez who warned me that already as a high school student, Enrique had the reputation of exhausting all his maths teachers, and told me that I should be very strong to control his incredible energy. And actually, I remember that during the years from 1985 to 1987, each morning when arriving at LJLL, after making 2 steps in the corridor, I saw at a distance Enrique saying "as a matter of fact, it turns out that..." or something of this type referring to our previous conversation that I partly forgot, since I had the strange habit of sleeping at night. I decided to do something very unusual at that time: on the pretext of the lecture notes, share the direction of research of Enrique with J.L. Lions. The plan worked perfectly: although initially Lions was a bit disturbed by the small southeast accent of Enrique, very soon he understood that Enrique never had enough work for his capabilities. The lecture notes were written, they were published, and as expectable Enrique immediately got some sharp results in the field, cf. e.g. [26]. To improve the plan I did one more thing: I introduced Enrique to another talented young colleague who followed regularly and very actively that course, namely Vilmos Komornik, with whom he immediately started to collaborate on some fundamental open questions raised by J.L. Lions, following a well established tradition which attracted a large audience. Their joint achievements were quite remarkable and have been highly appreciated at the international level, cf. e.g. the highly cited work [14].

\subsection{A very precocious recognition}

One afternoon, after his recognition by J.L. Lions but probably before the defense of the Ph.D. that was promising to approach the level of a habilitation, I remember asking Enrique, near the metro station of Jussieu, whether he was planning to look for a position in France or go back to Spain. He immediately answered that his parents were getting old and consequently he preferred to go back to Spain to remain not too far from them. By chance, some time later I happened to talk with Yves Meyer who just started to interact with J.L. Lions on some aspects of control theory. Yves Meyer had a very strong influence on Spanish mathematicians and, with a 3 lines firm recommendation letter from Lions, Enrique obtained very quickly a full professor position 
in Madrid. However, Enrique was not satisfied with doing classical academic research, he wanted his ideas to produce concrete results. We shall see that in the last section of this text.

\section{Some maturity ACHIEVEMENTS In CONTROL OF PARTiAL DIFFERENTIAL EQUATIONS}

After 1990, Enrique has been remarkably productive with numerous outstanding papers in control of partial differential equations. In particular ${ }^{1}$ :

- In [5], in collaboration with Caroline Fabre and Jean-Pierre Puel, Enrique Zuazua considers the nonlinear parabolic equation

$$
\left\{\begin{array}{l}
y_{t}-\Delta y+f(y)=u \chi_{\omega} \text { in }(0, T) \times \Omega \\
y=0 \text { on }(0, T) \times \partial \Omega \\
y=y^{0} \text { in } \Omega
\end{array}\right.
$$

In (3.1), $\omega$ is a nonempty open subset of $\Omega, \chi_{\omega}$ is the characteristic function of $\omega, u=u(t, x)$ is the control, and $f: \mathbb{R} \rightarrow \mathbb{R}$ and $y^{0}: \Omega \rightarrow \mathbb{R}^{n}$ are given functions. They assume the existence of $C>0$ such that

$$
f(y) \leqslant C(1+|y|), \forall y \in \mathbb{R} .
$$

Then they prove that the reachable set $\mathcal{R}$ defined by

$$
\mathcal{R}:=\left\{y(T) ; u \in L^{\infty}((0, T) \times \Omega)\right\}
$$

is dense in $L^{2}(\Omega)$. The proof relies on the minimization of a very cleverly chosen functional combined with a suitable fixed point argument. This strategy is very flexible. It has turned out to be very useful for many controllability issues for nonlinear control systems modeled by partial differential equations. It is a wonderful paper, which is already a classical one.

- In the breakthrough paper [28], Enrique considers the controllability of the semilinear wave equation

$$
y_{t t}-y_{x x}+f(y)=u \chi_{\omega}, \quad y(t, 0)=y(t, 1)=0,
$$

where the state is $\left(y, y_{t}\right)$ and the control is the function $u=u(t, x)$ and $\omega$ is a nonempty open interval of $(0,1)$. He proves the exact controllability (with an optimal time) when

$$
\lim _{|s| \rightarrow+\infty} \frac{f(s)}{s \log ^{2}(|s|)}=0 .
$$

The proof relies on a new strategy introduced by Enrique: it uses

- The Hilbert Uniqueness Method (HUM) due to J.-L. Lions,

- A fixed point argument (the Leray-Schauder fixed point here),

- Careful estimates on optimal controls for linearized control systems.

One cannot praise this paper too much: it is a paper which has strongly influenced a large number of people working in control of nonlinear partial differential equations. Let us point out that (3.4) is optimal. Indeed Enrique has also shown in this article that if $\omega \neq \Omega$ and if, for some $p>2$,

$$
\liminf _{s \rightarrow+\infty}(-f(s)) /\left(s \ln ^{p}(|s|)\right)>0
$$

\footnotetext{
${ }^{1}$ These are just examples. To be exhaustive on Enrique's papers in control of partial differential equations is an impossible target.
} 
blow-up phenomena cannot be avoided with the only help of the control.

- In [2], Enrique, in collaboration with Carlos Castro, studies the eigenvalues and eigenfunctions associated to the vibrations of a string with a rapidly oscillating bounded periodic density, that is the following eigenvalue problem

$$
\left\{\begin{aligned}
u^{\prime \prime}(x)+\lambda \rho\left(\frac{x}{\varepsilon}\right) u(x) & =0, \\
u(0)=u(1) & =0,
\end{aligned}\right.
$$

where $\rho$ is a periodic function and $\varepsilon$ is a small parameter (the size of the microstructure). They provide, among other things, all order correction formulas for the eigenvalues and the eigenfunctions when the size $\varepsilon$ of the microstructure is small compared to the wavelength $1 / \sqrt{\lambda^{\varepsilon}}$ of the eigenfunctions. Their method relies on a rigorous use of the classical asymptotic method WKB. This is the first time where the WKB method has been used in a rigorous way for singular perturbations problems. This result has turned out to be very useful for controllability problems. For example it has allowed them to get a very interesting result for the controllability of a wave equation with rapidly oscillating density.

- In [15], Enrique, in collaboration with Gilles Lebeau, studies the decay rates for solutions of the two and three-dimensional system of linear thermoelasticity in a bounded smooth domain with Dirichlet boundary conditions. They prove that there is no uniform exponential decay if there exist rays of geometric optics of arbitrarily large length that are always reflected perpendicularly or almost tangentially on the boundary. In dimension two they give a sufficient and (almost) necessary geometric condition for the uniform decay. They also give quite interesting applications of their results to controllability problems. This paper is a masterpiece. In particular it is a perfect illustration of the technical virtuosity of the authors.

- In [7], in collaboration with Enrique Fernández-Cara, Enrique considers a semilinear heat equation (in arbitrary dimension). They give a very interesting sufficient condition on the growth at infinity of the nonlinearity in order to have controllability. It turns that this condition allows blow-up in finite time if there is no control. This is a very surprising result, not at all expected. This is the first example of a nonlinear partial differential equation where one has blow-up in finite time without control and controllability when the control is there. The proof relies in particular on observability inequalities of heat equations with potentials. This important subject was already considered by Enrique in [6], in collaboration with Enrique Fernández-Cara, where a seminal global Carleman estimate is established for these equations. The sharpness of this estimate was proved in even dimension later on in [3] by Enrique, in collaboration with Thomas Duyckaerts and Xu Zhang. It is a major piece of work, for which the authors got the "Best article prize" of the Annales de l'IHP. It has been the source of inspiration for a vast literature.

- Singular perturbations for partial differential equations are hard problems. There are many works on this important subject without control. However the case of controlled partial differential equations is harder and has been much less studied. A pioneer work on this subject is the article [16] written by Enrique in collaboration with Antonio López and Xu Zhang. They consider the controlled wave equation

$$
\left\{\begin{array}{l}
\varepsilon y_{t t}-\Delta y=u \chi_{\omega} \text { in }(0, T) \times \Omega \\
y=0 \text { on }(0, T) \times \partial \Omega
\end{array}\right.
$$

In (3.5), as above, $\Omega$ is a nonempty bounded domain of $\mathbb{R}^{n}, \omega$ is a nonempty open subset of $\Omega, u=u(t, x)$ is the control. One has $\varepsilon>0$ and, as it is often the case, $\varepsilon \rightarrow 0^{+}$. Under suitable geometric conditions (related to the possibility to use the multiplier method) they prove the existence of converging controls as $\varepsilon \rightarrow 0^{+}$steering the control system (3.5) from a given state to 0 . As a consequence they get the null controllability of the heat equation. It is again a breakthrough work. It has opened the path to many other contributions.

- In pioneer papers, [4, 12, 13, 17-20, 24, 29, 30], Enrique studies the discretized version of observability inequalities for wave equations, heat equations, beam equations etc. For simplicity, let us consider only the wave equation. This is a long standing problem to understand how the finite time speed propagation 
of the wave equation, which imposes to have a large enough observation time $\left(T>T_{c}\right)$, can be seen on the corresponding semi-discretized version of the partial differential equation. One would expect that the constant of observability of the semi-discretized version of the wave equation do not blow-up as the meshsize tends to 0 if the time of observability $T$ is strictly larger than $T_{c}$. It turns out to be false as pointed by Roland Glowinski, Chin Hsien Li and Jacques Louis Lions in [8]. This is due to the appearance in the numerical scheme of spurious high frequencies, which interfere with the mesh, and imply that the group velocity of the semidiscrete model may vanish. This fact, that has been clearly analyzed and highlighted by Enrique Zuazua (partially together with his collaborators) in the above papers, is responsible for the failure of the uniform observability property. Enrique Zuazua has moreover studied some theoretical and numerical remedies to this bad behavior, suggested by R. Glowinski, such as Tychonoff regularization, filtering highfrequencies, multigrid methods, mixed finite elements, numerical viscosity, and, more recently, the very innovative use of nonuniform meshes with a way to construct them. He has studied and compared their efficiency, showing their numerical relevance (which lead in particular to sharp observability time). These are remarkable works which are very important both at practical and theoretical levels.

- In the beautiful and deep paper [1] Enrique in collaboration with Karine Beauchard, gives a precise decay rate for partially dissipative hyperbolic systems. Their proof relies on the introduction of a new original Lyapunov function, which is very cleverly chosen. This allows them to cover new situations. They also construct explicit Lyapunov functions. It is very useful for the feedback stabilization of control hyperbolic systems.

- In the seminal papers [21-23], Enrique Zuazua, in collaboration with Yannick Privat and Emmanuel Trélat, studies the optimal shape and location of the observation domain for heat and wave equations with random initial data. This leads to unexpected results with very different behaviors for these two different equations: a great stability for the heat equation, a great instability for the wave equation.

- Recently Enrique has been strongly involved in turnpike phenomena in the context of partial differential equations. The turnpike phenomena is the fact that for many interesting optimal control problem, if the time horizon is large enough, the optimal solution remains most of the time close to an optimal solution of a corresponding "static" optimal control problem. In particular in [25], in collaboration with Emmanuel Trélat and Can Zhang, Enrique establishes very general and powerful turnpike theorems which can be used for partial differential equations and indeed shows how one can use them for semilinear heat equations.

- At present, Enrique Zuazua's team is working on the use and applications of control methodologies to Machine Learning. In a recent article (arXiv:2008.02491), he, together with Carlos Esteve, Borjan Geshkovski and Dario Pighin, shows that the turnpike property holds for the Neural ODEs arising in Deep Supervised Learning when the parameters of the deep neural network are chosen so to optimize a criterion that involves a tracking term all along the evolution of the network. In another paper (arXiv:2104.05278) Enrique Zuazua, in collaboration with his Ph.D. student Doménec Ruiz-Balet, proves a fundamental result in the area, showing a strong simultaneous controllability result for the Neural Differential Equations, which provides a dynamic interpretation of the Universal Approximation Theorem. These very impressive results, allow to design more stable and robust neural networks, and open new promising perspectives at the interface between Control Theory and Machine/Deep Learning.

\section{FAR BEYOND PAPERWORK}

\subsection{Dissemination of scientific ideas and methods}

Enrique Zuazua has a strong experience in popularizing science. He received on two occasions the prize for scientific dissemination of the Spanish Applied Mathematical Society (SEMA). He had for many years a fortnightly radio show on the radio "Onda Vasca", a program that was highly appreciated by a wide audience.

\subsection{A strong international impact}

Enrique Zuazua was invited to prestigious international congresses (e.g. the International Congress of Mathematicians 2006, a sectional speaker in the section "Control Theory and Optimization"). He has been identified as 
a "Highly Cited Researcher" by ISI-Thomson. He received in 2007 the Julio Rey Pastor National Prize. Zuazua got two ERC advanced grants (NUMERIWAVES, 2010-2014 \& DYCON-Dynamic Control 2016-2021). He got the "Best article prize" of the Annales de l'IHP for [3]. He was invited during two consecutive years to give CIME lectures, which is exceptional. He is a member of the Academy Jakiunde, Eusko Ikaskuntzaren Zientzia, Arte eta Letren Akademia and of the Academia Europaea, Mathematics. He receives honorary doctorate from the University of Lorraine in 2014 and an important grant from the ANR (2016-2020). Enrique is presently a Humboldt Professor at Friedrich-Alexander-Universität (FAU) Erlangen-Nürnberg, a prestigious position. He assumes the chair in "Dynamics, Control and Numerics", which is designed to build a bridge between theory and practice and thus drive forward innovative, interdisciplinary research projects. As a result of Enrique's international activities he established strong stable collaborations with many foreign teams, as, for example, SCU in Chengdu and LJLL in Paris.

\subsection{A constructive leadership and mentoring}

His administrative experience and his leadership abilities are also remarkable. For example

- Enrique Zuazua has been and is still in the editorial board of many journals. In particular, he has been Corresponding Editor of SIAM J. Control Optimization and Editor-in-Chief of ESAIM: Control, Optimisation and Calculus of Variations. He is the founder of the collection of books SpringerBriefs in Mathematics, which aims is to publish contributions in Applied Mathematics, Finance, Statistics and Computer Science. He is presently Editor-in-Chief of Mathematical Control \& Related Fields.

- He has accepted to give numerous summer schools on control theory, which have stimulated research of many mathematicians. When he is a speaker at a summer school, one can be sure that there will a lot of audiences.

- In few years Zuazua built an excellent research center (Basque Center for Applied Mathematics, BCAM) for applied mathematics. BCAM permitted the return to Spain of many scientists.

- Enrique Zuazua always paid particular attention to the cooperation with developing countries, generating new opportunities for those that, without his help, probably would never had one.

Enrique Zuazua has been the advisor of over 28 Ph.D. students and more than 40 postdocs: https://www. genealogy.math.ndsu.nodak.edu/id.php?id=88528. All of them hold academic or industrial research positions and most of them have developed fruitful careers in Chile, China, France, Italy, Mexico, Romania, Spain, USA, etc. Among them:

\section{Ph.D. students:}

- Ana Carpio, Ph.D. 1993, Full Professor at Universidad Complutense de Madrid.

- Luz de Teresa, Ph.D. 1995, Full Professor at UNAM, Mexico; regular member of the Mexican Academy of Sciences since 2011 and President of Mexican Mathematical Society 2018-2020.

- Carlos Castro, Ph.D. 1996, Full Professor at Universidad Politécnica de Madrid.

- Sorin Micu, Ph.D. 1996, Full Professor at University of Craiova.

- Jaime Ortega, Ph.D. 1997, Full Professor at University of Chile.

- Liviu Ignat, Ph.D., 2006, Full Professor at University of Bucharest.

- Francisco Palacios, Ph.D. 2008, Senior Program Engineer en el 777 ALIT -Airplane Level Integration Team, Boeing, Seattle.

- Chuang Zheng, Ph.D. 2008, Associate Professor at Beijing Normal University.

- Alejandro Pozo, Ph.D. 2014, Researcher at Sherpa ai, Bilbao.

- Umberto Biccari, Ph.D. 2016, Assistant Professor at University of Deusto, Bilbao.

- Dario Pighin, Ph.D. 2020, Researcher at Sherpa ai, Bilbao.

- Borjan Geshkovski, Ph.D. 2021, Researcher at Sherpa ai, Bilbao. 


\section{Post-Docs:}

- Karine Beauchard, Full Professor at Ecole Normale Supérieure, Rennes, France; member of Institut Universitaire de France.

- Thomas Duyckaerts, Full Professor at Université Sorbonne Paris Nord, France; member of Institut Universitaire de France.

- Francesco Fanelli, Full Professor at Claude Bernard University Lyon 1, France.

- Dongnam Ko, Full Professor at Catholic University of Korea.

- Thibault Liard, Assistant Professor at Université de Limoges, France.

- Qi Lü, Full Professor at Sichuan University, China; invited speaker at ICM 2022.

- Arnaud Münch, Full Professor at Université de Clermont-Ferrand, France.

- Francesco Rossi, Full Professor at Università di Padova, Italy.

- Louis Tebou, Full Professor at Florida International University, USA.

- Xu Zhang, Full Professor at Sichuan University, China; invited speaker at ICM 2010.

- Julie Valein, Assistant Professor at Univ. Lorraine, France.

\section{A PROMising FUture}

The bridge between theory and practice was always the scientific "credo" (if any) of Jacques-Louis Lions which built an extremely robust and powerful group which celebrated its 50 years of existence in 2019. The main qualities which made this group not only robust, but more and more influential, are precisely the ones that Enrique decided to put forward in his approach to Mathematics: hardwork almost without limits, openness to new ideas, flexibility of mind and versatility of the methods in order to enlarge their field of applicability whenever possible. He used critical thinking to transform the problems into more understandable ones, a philosophy taught to us by Jacques-Louis Lions who always said, in substance: "if a problem looks too hard to be attacked upfront, try to encircle it and come back to the main point at the end". No doubt, it is with these intellectual choices that Enrique will use his present position as a Humboldt Professor to tackle new problems with direct implications in science and technology.

\section{REFERENCES}

[1] K. Beauchard and E. Zuazua, Large time asymptotics for partially dissipative hyperbolic systems. Arch. Ratl. Mech. Anal. 199 (2011) 177-227.

[2] C. Castro and E. Zuazua, Low frequency asymptotic analysis of a string with rapidly oscillating density. SIAM J. Appl. Math. 60 (2000) 1205-1233.

[3] T. Duyckaerts, X. Zhang and E. Zuazua, On the optimality of the observability inequalities for parabolic and hyperbolic systems with potentials. Ann. Inst. Henri Poincaré Anal. Non Linéaire 25 (2008) 1-41.

[4] S. Ervedoza, A. Marica and E. Zuazua, Numerical meshes ensuring uniform observability of one-dimensional waves: construction and analysis. IMA J. Numer. Anal. 36 (2016) 503-542.

[5] C. Fabre, J.-P. Puel and E. Zuazua, Approximate controllability of the semilinear heat equation. Proc. Roy. Soc. Edinburgh Sect. A 125 (1995) 31-61.

[6] E. Fernández-Cara and E. Zuazua, The cost of approximate controllability for heat equations: the linear case. Adv. Differ. Equ. 5 (2000) 465-514.

[7] E. Fernández-Cara and E. Zuazua, Null and approximate controllability for weakly blowing up semilinear heat equations. Ann. Inst. Henri Poincaré Anal. Non Linéaire 17 (2000) 583-616.

[8] R. Glowinski, C.H. Li and J.-L. Lions, A numerical approach to the exact boundary controllability of the wave equation. I. Dirichlet controls: description of the numerical methods. Jpn. J. Appl. Math. 7 (1990) 1-76.

[9] A. Haraux, Semi-linear hyperbolic problems in bounded domains. Math. Rep. 3 (1987) i-xxiv and 1-281.

[10] A. Haraux and E. Zuazua, Decay estimates for some semilinear damped hyperbolic problems. Arch. Ratl. Mech. Anal. 100 (1988) 191-206.

[11] A. Haraux and E. Zuazua, Super-solutions of eigenvalue problems and the oscillation properties of second order evolution equations. J. Differ. Equ. 74 (1988) 11-28.

[12] L.I. Ignat and E. Zuazua, Convergence of a two-grid algorithm for the control of the wave equation. J. Eur. Math. Soc. (JEMS) 11 (2009) 351-391.

[13] J.A. Infante and E. Zuazua, Boundary observability for the space semi-discretizations of the 1-D wave equation. ESAIM: M2AN 33 (1999) 407-438. 
[14] V. Komornik and E. Zuazua, A direct method for the boundary stabilization of the wave equation. J. Math. Pures Appl. 69 (1990) 33-54.

[15] G. Lebeau and E. Zuazua, Decay rates for the three-dimensional linear system of thermoelasticity. Arch. Ratl. Mech. Anal. 148 (1999) 179-231.

[16] A. López, X. Zhang and E. Zuazua, Null controllability of the heat equation as singular limit of the exact controllability of dissipative wave equations. J. Math. Pures Appl. 79 (2000) 741-808.

[17] A. Marica and E. Zuazua, Localized solutions and filtering mechanisms for the discontinuous Galerkin semi-discretizations of the 1-d wave equation. C. R. Math. Acad. Sci. Paris 348 (2010) 1087-1092.

[18] A. Marica and E. Zuazua, High frequency wave packets for the Schrödinger equation and its numerical approximations. $C$. $R$. Math. Acad. Sci. Paris 349 (2011) 105-110.

[19] A. Münch and E. Zuazua, Numerical approximation of null controls for the heat equation: ill-posedness and remedies. Inverse Probl. 26 (2010) 085018.

[20] M. Negreanu and E. Zuazua, Convergence of a multigrid method for the controllability of a 1-d wave equation. C. R. Math. Acad. Sci. Paris 338 (2004) 413-418.

[21] Y. Privat, E. Trélat and E. Zuazua, Optimal location of controllers for the one-dimensional wave equation. Ann. Inst. Henri Poincaré Anal. Non Linéaire 30 (2013) 1097-1126.

[22] Y. Privat, E. Trélat and E. Zuazua, Optimal shape and location of sensors for parabolic equations with random initial data. Arch. Ratl. Mech. Anal. 216 (2015) 921-981.

[23] Y. Privat, E. Trélat and E. Zuazua, Actuator design for parabolic distributed parameter systems with the moment method. SIAM J. Control Optim. 55 (2017) 1128-1152.

[24] L.T. Tebou and E. Zuazua, Uniform boundary stabilization of the finite difference space discretization of the 1-d wave equation. Adv. Comput. Math. 26 (2007) 337-365.

[25] E. Trélat, C. Zhang and E. Zuazua, Steady-state and periodic exponential turnpike property for optimal control problems in Hilbert spaces. SIAM J. Control Optim. 56 (2018) 1222-1252.

[26] E. Zuazua, Contrôlabilité exacte d'un modèle de plaques vibrantes en un temps arbitrairement petit. C. R. Acad. Sci. Paris Sér. I Math. 304 (1987) 173-176.

[27] E. Zuazua, Decay estimates for some semilinear damped hyperbolic problems. Asymptotic Anal. 1 (1988) 161-185.

[28] E. Zuazua, Exact controllability for semilinear wave equations in one space dimension. Ann. Inst. Henri Poincaré Anal. Non Linéaire 10 (1993) 109-129.

[29] E. Zuazua, Boundary observability for the finite-difference space semi-discretizations of the 2-D wave equation in the square. J. Math. Pures Appl. (9) 78 (1999) 523-563.

[30] E. Zuazua, Propagation, observation, and control of waves approximated by finite difference methods. SIAM Rev. 47 (2005) $197-243$. 